\title{
The US Army Corps of Engineers Scalable Approach to Estimating Loss of Life from Flooding
}

\author{
Jason Needham ${ }^{1, a}$, Woodrow Fields ${ }^{2}$, and William Lehman ${ }^{3}$ \\ ${ }^{1}$ P.E., USACE Risk Management Center, 609 2nd Street, Davis, CA 95616, USA \\ ${ }^{2}$ USACE Hydrologic Engineering Center, 609 2nd Street, Davis, CA 95616, USA \\ ${ }^{3}$ USACE Hydrologic Engineering Center, 609 2nd Street, Davis, CA 95616, USA
}

\begin{abstract}
Although flooding can lead to many types of severe consequences, the primary objective of the US Army Corps of Engineers (USACE) dam and levee safety programs are to manage risk to the public who rely on those structures to keep them reasonably safe from flooding. Thus, reducing the risk associated with loss of life is paramount. USACE employs a scalable approach to estimating loss of life, where the goal is to invest an appropriate amount of resources to answer the question at hand. Given the large number of dams and levees within the USACE portfolio (over 700 dams and 15,000 miles of levees), a "one size fits all" approach does not work. Screening-level risk assessments are carried out to initially characterize risk. That initial risk characterization informs prioritization of additional efforts such as interim risk management activities and more detailed risk assessments. More detailed risk assessments inform investment in long-term risk reduction measures, which include analysis and selection of major modification activities and implementation of non-structural measures including emergency management and evacuation planning. This paper describes the USACE scalable approach for estimating loss of life from flood events.
\end{abstract}

\section{Introduction}

To effectively manage a portfolio of dams or levees, estimates of potential loss of life due to flooding (breach or non-breach related) are needed for the following purposes:

- To rapidly assess the portfolio and characterize relative risks for initial prioritization [1,2],

- To evaluate existing and residual risks against tolerable risk guidelines [3],

- To assess proposed risk-reduction measures, including non-structural approaches such as more effective warning and emergency management activities.

Estimation of the magnitude of life loss resulting from a flood requires consideration and estimation of the following factors:

- Flood severity, which can be described by extents of inundation, depths, velocities, and arrival time;

- Exposed population; which is the portion of the population at risk (PAR) still in the area when the flood water arrives. This requires an understanding of the warning and evacuation effectiveness. (Figure 1);

- Shelter type, which accounts for potential safety provided by the location where exposed population is located (wooden home, concrete high-rise, out in open, etc.), and;
- Fatality Rates, which define the likelihood that people subjected to the flooding will die given the flood characteristics and shelter type.

The full consideration of all these factors is a complex problem that requires detailed modelling of the physical processes (breach characteristics and flood routing), the warning and evacuation planning and processes, human decision-making, and performance of transportation systems (evacuation) and buildings under flood loading.

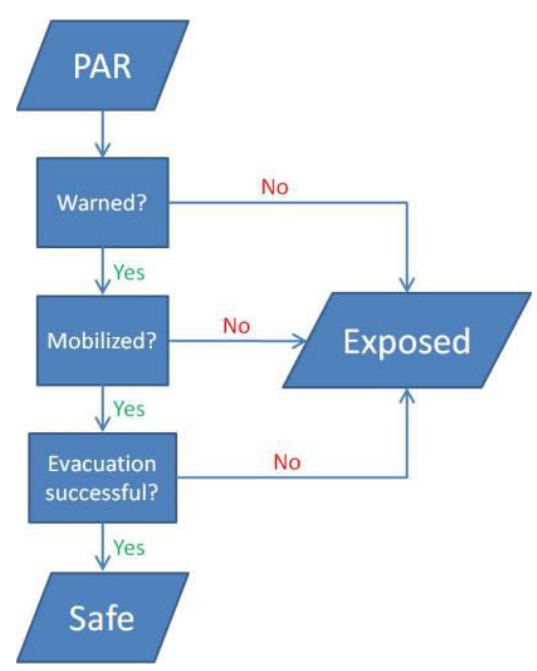

Figure 1. Relationship between PAR and Exposed Population

\footnotetext{
${ }^{\mathrm{a}}$ Corresponding author: jason.t.needham@usace.army.mil
} 
This papers describes a range of practical approaches to this complex problem that provide life-loss estimates for use in all levels of risk assessment within USACE.

\section{Categories of Risk Assessments}

Methods for estimating potential loss of life from flooding that are used to support the USACE Dam and Levee Safety program risk-informed portfolio management process can be divided into two broad application categories: Screening and Detailed. Generally, screening level assessments generate results that are appropriate for informing initial risk classification and prioritization of additional studies for the portfolio. Detailed or higher level assessments can be used to verify findings from screening level assessments prior to undertaking major modification activities as well as informing the decision making process for the appropriate risk reduction approach. The next two sections describe the life loss estimation methods used by USACE to support both screening and detailed risk assessments.

\section{Screening-level Life Loss Estimation}

Within the context of portfolio management, the primary purpose of a screening-level risk assessment is to categorize and prioritize additional investments. Investments based on screening-level assessments primarily include additional, more detailed, studies and interim risk reduction measures. Therefore, it is proper for a screening assessment to "err on the side of caution". In other words, the tools and decision-making process used within the screening context should result in classifications that, if wrong, when looked at closer will show that the understanding of risk is lower than identified through the screening process. The result of this approach is that some projects will be given high priority for more detailed assessments when ultimately not warranted. However, the consequence of not using the "err on the side of caution" approach is that some high-risk projects could go unattended for years; a much greater issue to be sure.

The primary inputs required for the life loss estimates provided by the USACE levee screening process [1] include a delineation of the area protected by the levee (leveed area), digital terrain model for that leveed area, maximum and minimum top-of-levee elevations, and day and night-time population within the leveed area. Their use is described below.

\subsection{Flood Severity for Screening Assessments}

USACE developed a Levee Screening Tool (LST) to assist with the levee screening process. A simplified breach flow spreading approach is included in the LST that estimates the worst case potential depth of water in the leveed area due to levee breach. The worst case depth at any location in the leveed are is estimated using a simple bathtub approach. The user estimates the maximum water surface elevation that can be achieved in the leveed area, either by defining the maximum bounding elevation of the terrain (rim of bathtub) or the maximum water surface elevation that can be achieved due to hydrologic considerations. For leveed areas where a bathtub effect would not occur (water would flow through rather than ponding), a 0.6 meter ( 2 feet) overland flow depth is applied across the leveed area.

\subsection{Exposed Population for Screening Assessments}

The exposed population is computed as the number of persons that do not safely evacuate the leveed area and therefore are exposed to flooding resulting from breach or overtopping of the dam or levee in question. Exposed population is typically computed as a fraction of the total Population at Risk (PAR) in the potentially impacted area.

For a screening assessment, exposed population is computed in the LST based inputs for Population at Risk and a series of evacuation effectiveness factors that are entered by the team completing the screening. Population at risk (PAR) estimates are based on the number of persons within the leveed area. Existing data from nationally available sources are used to determine the PAR estimate.

The factors used to estimate evacuation effectiveness include:

1. Community Awareness,

2. Evacuation Planning,

3. Warning System Effectiveness,

4. Transportation System Congestion, and

5. Warning Time

The team applying the LST uses the available emergency action plans, local knowledge, and other information to make an assessment of above factors. The factors are qualitative in nature, meaning the team enters either "Acceptable, Minimally Acceptable, or Unacceptable" for each of the first four factors. Guidance is provided to the teams that defines what constitutes an appropriate rating in each category. The LST then converts those qualitative entries into quantitative estimates of evacuation effectiveness.

Since the LST risk estimate covers a prescribed range of potential failure modes, life loss estimates are needed for each. Performance modes related to failure and breach of the levee prior to overtopping assume that adequate warnings are not issued prior to failure. Overtopping events assume that adequate warnings are issued prior to overtopping and breach. The evacuation effectiveness factor is adjusted to account for the different warning scenarios.

\subsection{Shelter Type and Fatality Rates for Screening Assessments}

Using research by Jonkman [4], fatality rates (FR) are computed for different depths in the protected area. 
Jonkman's research does not explicitly account for shelter type, rather they are built into the empirical data used to derive his fatality rate relationships. Jonkman's research shows that the protected area can be divided into two zones for estimating fatality rates, the breach zone and the remaining zone. The breach zone, which is defined as an area near the breach with a high value for depth times velocity $\left(\mathrm{hv} \geq 5 \mathrm{~m}^{2} / \mathrm{s}\right)$, is not used in the LST due to the added complexity required to identify breach location, estimate local velocities and depths in the breach zone and the inherent uncertainty in predicting the breach location in a screening assessment. The rest of the protected area is part of the remaining zone.

His research on life loss in New Orleans during Katrina resulted in fatality rate a relationship as a function of depth for the remaining zone. Since shelter types are not explicitly considered, using the fatality rate computes from that research in an area where the built inventory (residences, buildings, etc) are significantly different than New Orleans could introduce significant error.

\section{Detailed Life Loss Estimation}

The goals of a detailed life loss estimate can be generally defined as follows:

1. Verify or modify screening-level understanding of potential life loss and risk;

2. Provide a life loss estimate that can be used by decision-makers to measure against agency tolerable risk guideline to inform remediation efforts;

3. Understand what is driving the life loss so that risk reduction measures can be formulated to reduce risk to meet the agency tolerable risk guidelines.

USACE utilizes the LifeSim methodology for all estimates of life loss beyond screening-level. This methodology is contained in HEC-FIA as well as HECLifeSim. HEC-FIA contains a simplified evacuation modelling approach where the user either provides the amount of time required for inhabitants of each structure to evacuate to safety or provides a hazard boundary in the form of a polygon shapefile. If a hazard boundary is provided, HEC-FIA determines the shortest straight-line distance from a structure to the hazard boundary and applies a nominal evacuation speed along that line to estimate the amount of time required to evacuate. The effect of traffic jam potential must be accounted for implicitly by the choice of the nominal evacuation speed. If the loss of life for a study is highly dependent on evacuation efficiency, including the effects of traffic congestion, application of the full version of LifeSim should be considered as it contains a more detailed evacuation model.

The full version of LifeSim is applied to support risk assessments within USACE when the simplifying assumptions inherent in HEC-FIA result in too much uncertainty in the life loss estimate. Also, given the detailed evacuation modelling available in LifeSim, it is also applied to assist with evacuation planning.

\subsection{Flood Severity for Detailed Assessments}

LifeSim requires hydraulic inputs from an exterior hydraulic modeling system. For detailed life loss assessments, an unsteady hydraulic modeling approach (one- dimensional or two-dimensional as appropriate) is used to develop the inundation boundaries, arrival times, depths, and velocities necessary to estimate potential loss of life.

To support a detailed risk assessment, a range of flood scenarios are modelled. This range of scenarios includes breach at different pool or river levels as well as nonbreach scenarios where flooding could lead to potential life loss. Other important considerations in hydraulic modeling include breach parameters and coincident hydrologic conditions (reservoir inflow, tributary flows and stages, status of interior drainage system, etc). Often these additional consideration are handled through sensitivity and uncertainty analysis, and a range of potential life loss results are provided for each scenario rather than a single value.

\subsection{Exposed Population for Detailed Assessments}

As mentioned under the screening assessment section, the exposed population represents the portion of the population at risk that do not safely evacuate the leveed area, and therefore are exposed to flooding resulting from breach or overtopping of the dam or levee in question (refer

to

Figure 1). Figure 2 illustrates the warning and evacuation timeline within the context of a detailed assessment, which starts when the emergency management agency responsible for alerting the population at risk receives notification that a hazard is imminent, and ends when the population at risk takes the recommended protective action.

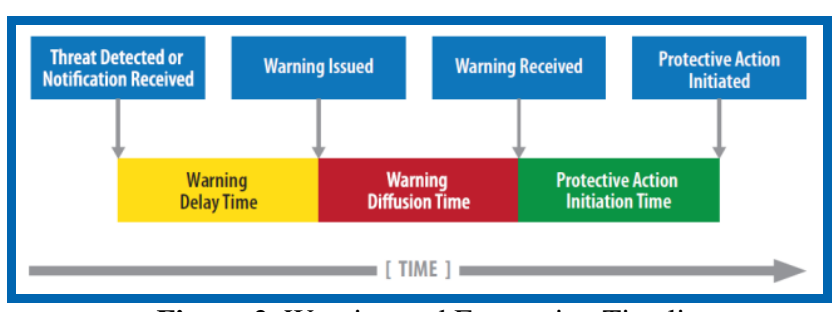

Figure 2. Warning and Evacuation Timeline

Three primary time components make up the warning and evacuation timeline:

1. Warning delay time

2. Warning diffusion time

3. Protective action initiation time

Finally, if the protective action is to evacuate by vehicle or on foot, the amount of time it takes to reach a safe location is also an important aspect of the warning and 
evacuation timeline, and is discussed in the section along with the three items listed above.

\subsubsection{Warning delay time}

The warning initiation time is defined as the point in time when an official alert or warning is first sent out from the responsible emergency management agency to the population at risk. Prior to warning initiation, there is a decision-making process that occurs within the emergency management agency. The amount of time that decision takes to make is called the warning issuance delay time, and covers the time from when the agency learned about the impending hazard and when they flip the switch on whatever warning systems they have at their disposal.

Factors that most influence the warning initiation delay time are whether the emergency management agency has a warning plan and procedures written down, and whether they have a warning decision matrix in place. A warning decision matrix describes all the possible issues, or evidence of potential issues (e.g. increased seepage under levee), what that means in terms of a potential flood threat, and what message should be sent to the population at risk under that scenario. If all of these items have been thought through ahead of time, then the process will be much more efficient during an actual event.

\subsubsection{Warning diffusion time}

The speed at which a warning spreads (warning diffusion) varies between communities and events. Some dissemination channels reach more people more quickly than others, and some types of people are easier to reach than others.

Warnings can come from a formal emergency management system, through informal communication processes, or directly from cues in a person's environment. It can be an alert (signal) or a notification (message). Formal alerts and warnings can come via a number of different communication channels involving both new (e.g, cell phone or internet) and established (e.g., TV, radio, siren, route alert) technologies. Each type of channel has strengths and weaknesses, such as the speed of dissemination, ability to convey information, and susceptibility to failure.

The receipt of a warning is also influenced by the characteristics of the people for whom the message is intended. These characteristics include the activities that people are engaged in, where they are located, the time of day, reception impediments (visual or hearing impairment), and the personal resources available. Warning diffusion rates are also the impacted by the ability to receive an alert or warning (e.g. if it is nighttime or the intended recipients have hearing impediments.)

The warning dissemination process is modelled in LifeSim through the use of warning diffusion curves. A warning diffusion curve defines the relationship between time from warning issuance and the percentage of the population at risk that has received that warning. Figure 3 displays a moderately fast warning diffusion curve with uncertainty as well as the maximum and minimum bounds are warning diffusion based on available research [5]. As you can see, warning diffusion speed can vary greatly.

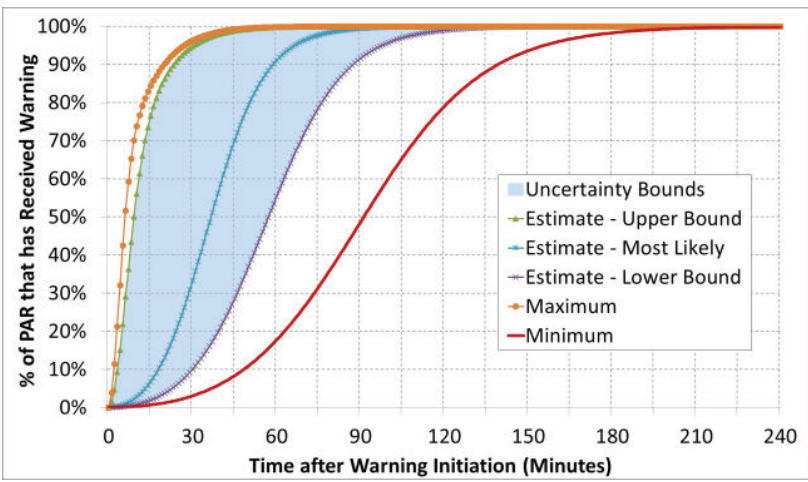

Figure 3. Example Warning Diffusion Curve

Warning diffusion relationships should be estimated based on discussions with local emergency managers. In many cases it will be necessary to define different warning diffusion curves for different sub-populations. USACE uses a consequence elicitation process to determine diffusion curves. That elicitation process follows guidance from social sciences on the questions that should be asked of the emergency managers and methods for taking their answers and selecting appropriate diffusion curves. Contact the USACE Risk Management Center for an up-to-date consequence elicitation guidance.

\subsubsection{Protective action initiation time}

Protective Action Initiation (PAI) delay time is defined as the amount of time between when a warning is received by a person and when that warned person takes the recommended protective action (e.g. leaving their structure). Like warning diffusion times, PAI delay times are modelled in LifeSim through the use of PAI curves. These PAI curves contain two important pieces of information for determining the number of people that have evacuated their structures when the flood arrives: (1) the percentage of warned people that mobilize over time; and (2) the maximum mobilization percentage.

The maximum mobilization percentage defines the highest percentage of people that would attempt to leave the potentially inundated area if time was available. One hundred percent minus the maximum mobilization percentage yields the percentage of people that are either unable or choose not to mobilize after receiving the warning. Like warning diffusion relationships, mobilization curves are defined based on results of consequence elicitation. USACE follows a consequence elicitation process that was developed by social scientists familiar with the primary factors that influence mobilization based on available research.

The range of mobilization curves defined in that research are shown in Figure 4. It is recognized that the life loss estimate is highly dependent on the mobilization 
information provided to LifeSim, and that the actual mobilization decision process contains many contributing factors that are highly uncertain. The elicitation effort results in a most likely curve, with an upper and lower bound that all fall within the range of the curves shown in Figure 4.

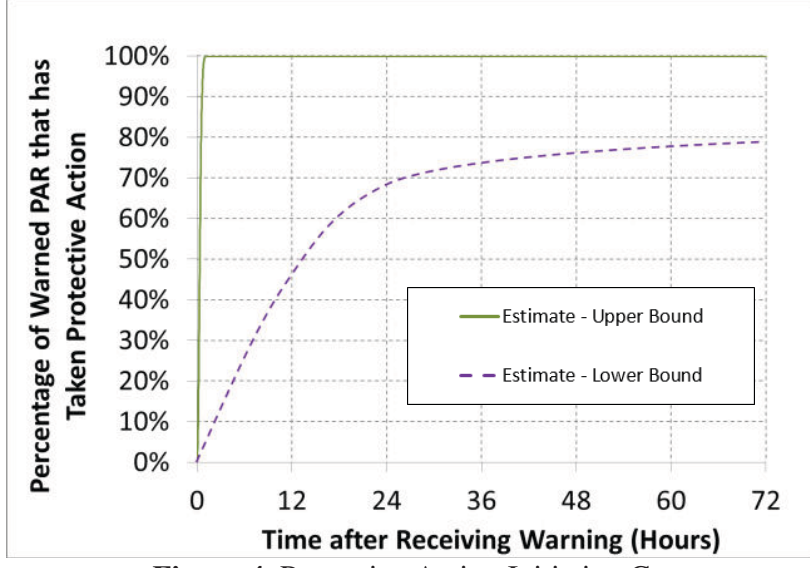

Figure 4. Protective Action Initiation Curves

\subsubsection{Evacuation Time}

LifeSim uses an agent-based approach to track individuals throughout the warning and evacuation process. It also contains a traffic simulation engine to simulate the evacuation process that allows vehicles to interact with other vehicles and the hazard. The computation engine offers an effective estimation of population re-distribution during the evacuation process. LifeSim was developed with an uncertainty sampling approach. By sampling uncertain parameters and running the model iteratively, LifeSim is capable of producing a distribution of results to better inform a risk assessment.

It is important to note that LifeSim is simulating the entire warning, mobilization, and evacuation process. The process can be illustrated with the following example.

Consider a single family home containing three people that is located in the study area for a given simulation. Once a flood warning is issued by emergency managers, it takes some amount of time (warning diffusion) before a resident in the home receives the warning. Once the warning is received and understood at the home, the family members will take some time (protective action initiation delay) to gather their belongings and prepare for leaving their home. The family then leaves their home in a car containing three people. On the road, they potentially interact with other vehicles and flood waters. For example, they may encounter a traffic jam at a freeway on-ramp and choose a different route out of the hazard area. Or they may choose to stay in the traffic jam, hoping that it will clear up. If the family reaches a road that is currently flooded they can attempt to turn around and go in a different direction. If the family gets caught on a flooded road, the survival of each family member is dependent on their state (e.g. age) and the hydraulic conditions at their location. All of these interactions are simulated during
LifeSim model iteration. Because warning initiation times, warning diffusion curves, and protective action initiation delay curves, are sampled from a range of possibilities, the same family may take longer to get warned or choose to remain in their home during the next model iteration.

Simulation results for existing conditions and alternatives can be visualized using LifeSim animation capabilities. In the LifeSim results animation image shown in Figure 5, structures and cars caught (inundated) by floodwaters are red. Yellow structures indicate that a warning was received but the people inside haven't started evacuating yet, and brown structures indicate that a warning hasn't been received yet. Blue cars represent people mobilizing on roads, and are tracked throughout the study area based on traffic simulation algorithms.

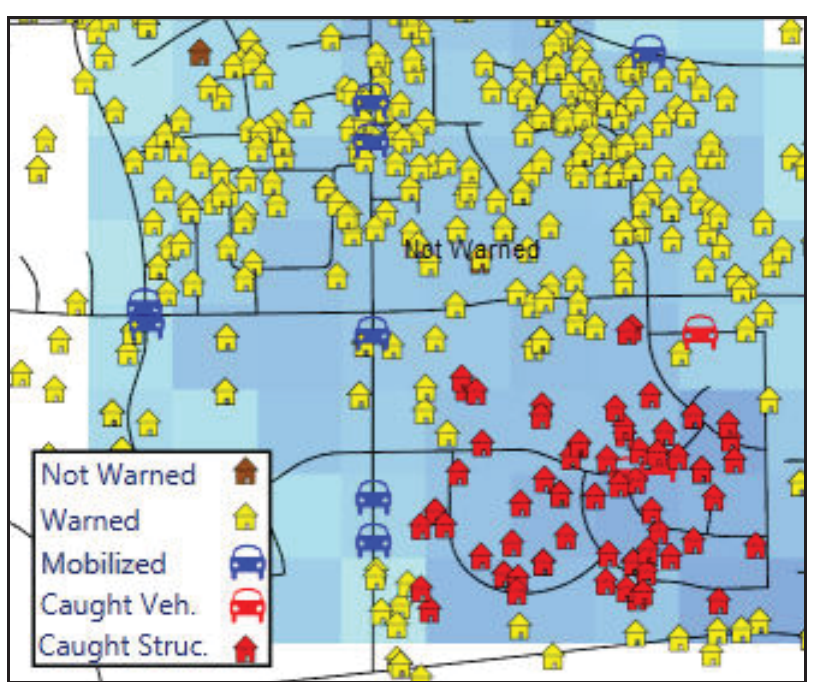

Figure 5. LifeSim Animation Results

By tracking individual people and their movements, LifeSim can help identify where people are most at risk of losing their lives, whether it is on roads or in structures. We can now pinpoint the locations of greatest potential life loss, which is useful when developing alternative project formulations. For example, a simulation may show that life loss on a particular road is significant. LifeSim allows for a detailed analysis of a range of alternatives based on both structural and nonstructural measures for reducing potential life loss. Nonstructural measures to reduce life loss could include raising or closing at risk road embankments and increasing road capacities to reduce congestion. A nonstructural alternative could also consist of increasing the warning time through better warning issuance and community awareness.

\subsection{Shelter Types and Fatality Rates for Detailed Assessments}

After the warning, mobilization, and evacuation process has been simulated, LifeSim categorizes those people that are exposed to flooding into three flood lethality zones. The lethality zone categorization is a function of shelter effectiveness and the depths and velocity of flooding at that location. Shelter effectiveness 
is determined based on the construction of the building in question and associated stability criteria. For example, if located in a wood building, the shelter effectiveness is determined based on the depths and velocity of flooding at that location, the stability criteria for wooden structures, and the height of the structure (if the structure survives but depth exceeds the height of the structure, the shelter effectiveness is negligible). Another example would be if someone is caught out in the open while evacuating on foot. In that scenario, the shelter effectiveness would be based the relationship between the depths and velocities at that location and human stability criteria.

Flood lethality zones distinguish physical flood environments where historical rates of life loss have distinctly differed. Three flood zones are physically defined by McClelland and Bowles [2002] by the interplay between available shelter and local flood depths and velocities, summarized as follows:

- Chance zones in which flood victims are typically swept downstream or trapped underwater and survival depends largely on chance; that is, the apparently random occurrence of floating debris that can be clung to, getting washed to shore, or otherwise finding refuge safely. Historical fatality rates range from about 50 percent to 100 percent, with an average rate of about 90 percent.

- Compromised zones in which the available shelter has been severely damaged by the flood, increasing the exposure of flood victims to violent floodwaters. An example might be when the rooms inside a building experience rapidly-moving shoulder-height flood water. Historical fatality rates range from zero to about 50 percent, with an average rate of about 10 percent.

- Safe zones are typically dry, exposed to relatively quiescent floodwaters, or exposed to shallow flooding unlikely to sweep people off their feet. Examples might include the second floor of residences and sheltered backwater regions. Historical fatality rates are virtually zero.

By focusing on the flood characteristics and shelter effectiveness where people are located, LifeSim removes the connection between potential loss of life from a specific dam or levee breach, and makes it applicable for any type of flood scenario.

As with the other inputs to LifeSim, the understanding of fatality rates, which is the likelihood that someone exposed to a certain severity of flooding will die, is highly uncertain. Therefore, fatality rates are also provided in the form of distributions, which are sampled in each iteration.

\section{Concluding Remarks}

There have been substantial advancements in the field of flood-related life loss assessment over the last decade.
These advancements are not only in methodology (e.g. improved evacuation modelling), but also in the application of methodology (e.g. better understanding warning and evacuation processes, which provides better inputs to many methodologies). However, even with these improvements, any estimate of potential loss of life carries significant uncertainty along with it. Regardless of the method used, it is important to characterize the uncertainty that comes along with it.

Also, calibration and validation of simulation-based life loss models, like HEC-FIA or LifeSim, is a significant challenge (if not impossible). Calibration of a specific model, which is the process of verifying that results match well with field measurements for a range of magnitudes of events, is obviously not possible. Validation of a methodology, which demonstrates that method can provide reasonable forecasts of potential life loss, can be achieved in some fashion by using that methodology to model historic events. However, the data required to validate a methodology using a historic event is rarely available. Understanding where people are located at the time of an event, when they were warned, how they were warned, and the decisions they made related to if and how they evacuated is practically impossible to obtain. Regardless, USACE has successfully validated the methods described herein with specific events including the Katrina hurricane in New Orleans and Malpasset Dam Failure in France. The validation process shows that the standard approach used by USACE, including assumptions on various inputs that are not available from the historic record, provides results very close to what actually happened.

\section{References}

1. Margo, D., Harkness, A., and Needham, J. (2009). Levee Screening Tool. United States Society on Dams Conference, Nashville, April 20-24.

2. McClenathan, J.T. (2010). Update for Screening Portfolio Risk Analysis for U.S. Army Corps of engineers Dams. Proceedings of the 2010 USSD Annual Lecture, Sacramento, CA, April 12-16.

3. Munger, D.F., Bowles, D.S., Boyer, Davis, D.W., Margo, D.A., Moser, D.A., Regan, P.A, and Snorteland, N.J. (2009). Interim Tolerable Risk Guidelines for US Army Corps of Engineers Dams. Proceedings of the 2009 USSD Annual Lecture, Philadelphia, Pennsylvania, March.

4. Jonkman, S.N. (2007). Loss of life estimation in flood risk assessment- theory and applications. $\mathrm{PhD}$ thesis, Delft University.

5. Sorensen, J. and D. Mileti (2014). First Alert and/or Warning Issuance Time Estimation for Dam Breaches, Controlled Dam Releases, and Levee Breaches or Overtopping. Draft paper prepared for U.S. Army Corps of Engineers. Institute for Water Resources, Risk Management Center. Davis, CA. 